\title{
Power Harvesting Capabilities of SHM Ultrasonic Sensors
}

\author{
Christophe Delebarre, ${ }^{1}$ Thomas Sainthuile, ${ }^{1}$ Sébastien Grondel, ${ }^{1}$ and Christophe Paget ${ }^{2}$ \\ ${ }^{1}$ IEMN, UMR CNRS 8520, Département OAE, Université de Valenciennes et du Hainaut Cambrésis, \\ Le Mont Houy, 59313 Valenciennes Cedex 9, France \\ ${ }^{2}$ Airbus Operations Limited, Bristol BS99 7AR, UK \\ Correspondence should be addressed to Christophe Delebarre, christophe.delebarre@univ-valenciennes.fr
}

Received 19 January 2012; Accepted 9 March 2012

Academic Editor: Mickaël Lallart

Copyright (๑) 2012 Christophe Delebarre et al. This is an open access article distributed under the Creative Commons Attribution License, which permits unrestricted use, distribution, and reproduction in any medium, provided the original work is properly cited.

\begin{abstract}
The aim of this work is to show that classical Structural Health Monitoring ultrasonic sensors may provide some power harvesting capabilities from a wide variety of vibration sources. In other words, the authors developed an integrated piezoelectric energy harvesting sensor capable of operating a dual mode, that is, carrying out vibration power harvesting and Structural Health Monitoring. First, vibrations signals of an A380 aircraft recorded during different phases of flight are presented to show the need of a wideband piezoelectric energy harvester. Then, the voltage response of a piezoelectric power harvester bonded onto an aluminium cantilever plate and excited by an electromechanical shaker is measured. A finite element model of the energy harvester system is also presented. This model provides the voltage response of the harvester due to a mechanical excitation of the host structure and allows a better understanding of the energy harvesting process. In many cases, a good agreement with the experimental results is obtained. A power measurement also showed the ability of piezoelectric SHM sensors to harvest power over an extended frequency range present in spectra collected in aircrafts. This result could lead to numerous applications even though this kind of power harvester sensor has been initially designed to operate onboard aircrafts.
\end{abstract}

\section{Introduction}

Energy harvesting, or scavenging as it is frequently called, provides new opportunities for sensor manufacturers in applications that would otherwise have difficulty obtaining a reliable power source. Adding a power source implies the need for replacements as well as maintenance procedures involving costs increase. Various techniques exist in order to carry out energy harvesting. They are based on light or temperature difference [1], radio frequency [2], inductive coupling, wind energy [3], and mechanical vibration conversion [4-8]. For these purposes, several pieces of equipments are used, such as MicroPelletier, wind turbine, RF energy converter, solar panel, and piezoelectric sensors.

In this paper, the authors study the feasibility of developing a Structural Health Monitoring (SHM) system having a double functionality, that is, carrying out SHM tasks but also energy harvesting in order to be fully autonomous. This SHM system has been initially built to perform damage assessment of aeronautic structures using well-known techniques like the Selective Lamb Mode Technique [9], Acoustic
Emission Monitoring [10, 11], and Lamb waves interaction $[12,13]$. Consequently, the energy harvesting technology that has been retained is based on the conversion of mechanical vibration into electrical power using piezoelectric sensors. This technology seems well suited for the SHM system since various mechanical energy sources are generated by transportation vehicles during their displacement. On one hand, ground transportation can provide vibrations coming from the mechanical coupling between the road or track and the body of the vehicle and also from the engine. On the other hand, airplane vibrations are mainly due to aerodynamic noise and engines vibrations. However, in these transportation applications, the source of ambient vibration energy may fluctuate and the spectral components of the different sources of vibration that can be harvested may also change with the environmental conditions.

Furthermore, the initial spectrum of the vibration source may be seriously modulated by the natural frequencies of the entire structure. This fact induces the necessity of developing wideband piezoelectric energy harvesters since the Power 


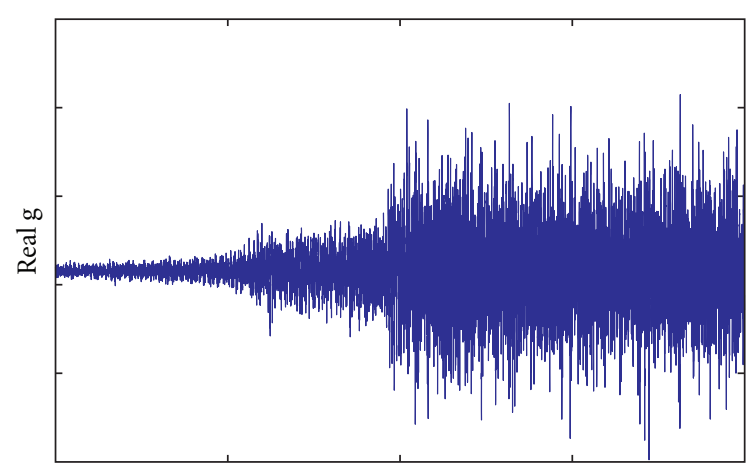

Time

Figure 1: Takeoff vibration signal of an A380.

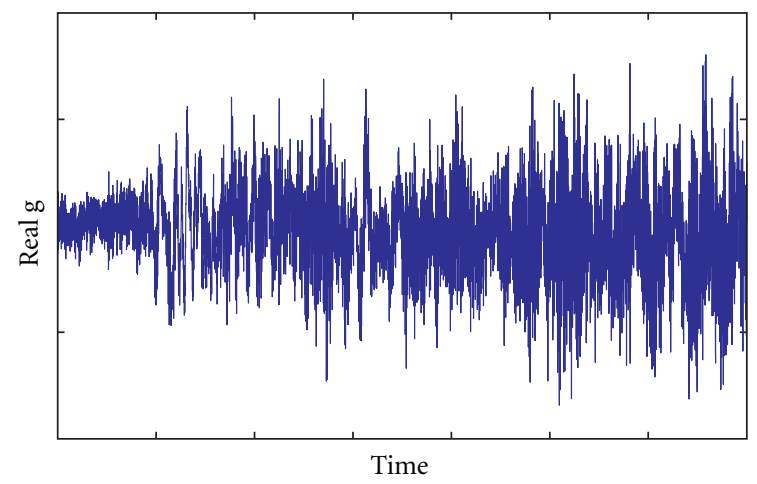

FIGURE 2: Landing vibration signal of an A380.

Spectrum Density (PSD) of such vibration sources clearly shows that the energy is spread all over the spectrum.

However, even if the energy can be seen as wideband, its main part remains concentrated in the low-frequency part of the spectrum. This is the reason why cantilever beam energy harvesters have been studied and developed in the $50-300 \mathrm{~Hz}$ band [5-8]. However, these harvesters are frequency tuned devices due to the mass used to amplify the displacement of the piezoelectric beam at a given frequency. As a result, they provide a good electromechanical conversion rate in a very narrow frequency band.

Figures 1 and 2 show several vibrations recorded by a $250 \mathrm{~Hz}$ bandwidth accelerometer during the landing and take off phases of an A380 aircraft for different position of the sensors [Airbus sources]. The sensor used for the takeoff was bonded near the wing/fuselage junction, while the sensor used for landing was bonded at the extremity of the wing.

One can see that the vibration amplitudes and frequencies vary depending on the phases of flight and of the location on the aircraft. This shows the importance of the location of the energy harvester since one has to ensure that the bonding locations of these sensors are actually providing vibration displacements. Figure 3 gives the Wigner-Ville transform of the landing signal. During landing, other vibrations with higher energy level occur. Note that the bandwidth of the accelerometer shows that the frequency components of the spectra are not significant after $250 \mathrm{~Hz}$.

All these measurements show the need of wideband frequency power harvesting. Moreover, when using a SHM

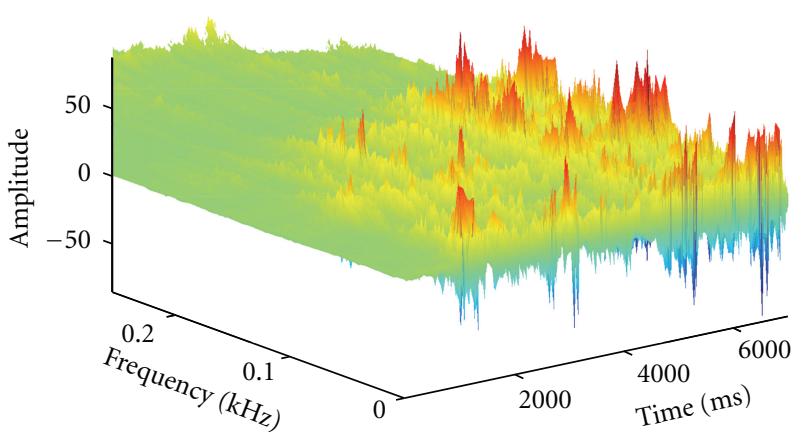

Figure 3: Time-frequency analysis of the vibrations for A380 aircraft (landing phase).

sensor to carry out the energy harvesting task, the Thevenin model can be used to represent the energy harvester. The sensor can be seen by the electrical load like a dipole constituted by a voltage generator in serial with its internal output impedance. In that case, optimization of the harvested power could be treated by maximising the static voltage value for the generator and minimising the value of the output impedance. Consequently, the voltage response of the sensor is of importance as it will be directly related to the power harvested. Developing various models of the sensor response to a mechanical vibration will allow the evaluation of the sensor's capabilities to detect a particular type of vibration $[14,15]$ and to harvest energy [16].

Consequently, the authors are investigating the ability of the SHM sensors to harvest energy from a wide variety of vibration sources all over the frequency spectrum while keeping their SHM capabilities. To start with, an experiment will be carried out. Wideband mechanical vibrations will be generated throughout a structure instrumented with several SHM sensors. Then, the voltage response over an extended frequency range will be evaluated. In order to obtain a better understanding of the energy harvesting phenomena, a numerical model based on the Finite Element Method (FEM) will be developed. It will simulate the experimental setup and will model each part of the process allowing the evaluation of the SHM capabilities for a mechanical excitation as well as the study of the vibration propagation. Finally, a power estimation will be conducted.

\section{Experimental Phase}

2.1. Experimental Setup. In order to measure the electrical power which could be harvested using the different sizes of the harvesters, an experimentation setup has been built. It consists of an aluminium plate $(500 \mathrm{~mm} * 300 \mathrm{~mm} * 3 \mathrm{~mm})$ having one fixed edge, while the others remained free, resulting in a cantilever configuration. The plate has been instrumented with four SHM piezoelectric energy harvesters. The SHM system of this study uses Lamb Waves within the structure to detect and locate damages. To generate these Lamb Waves in aluminium plate-like structures, piezoelectric elements working around their radial resonant frequency and polarized along their thickness have to be used. That is why small discs with a 20-millimetre diameter and a thickness of 


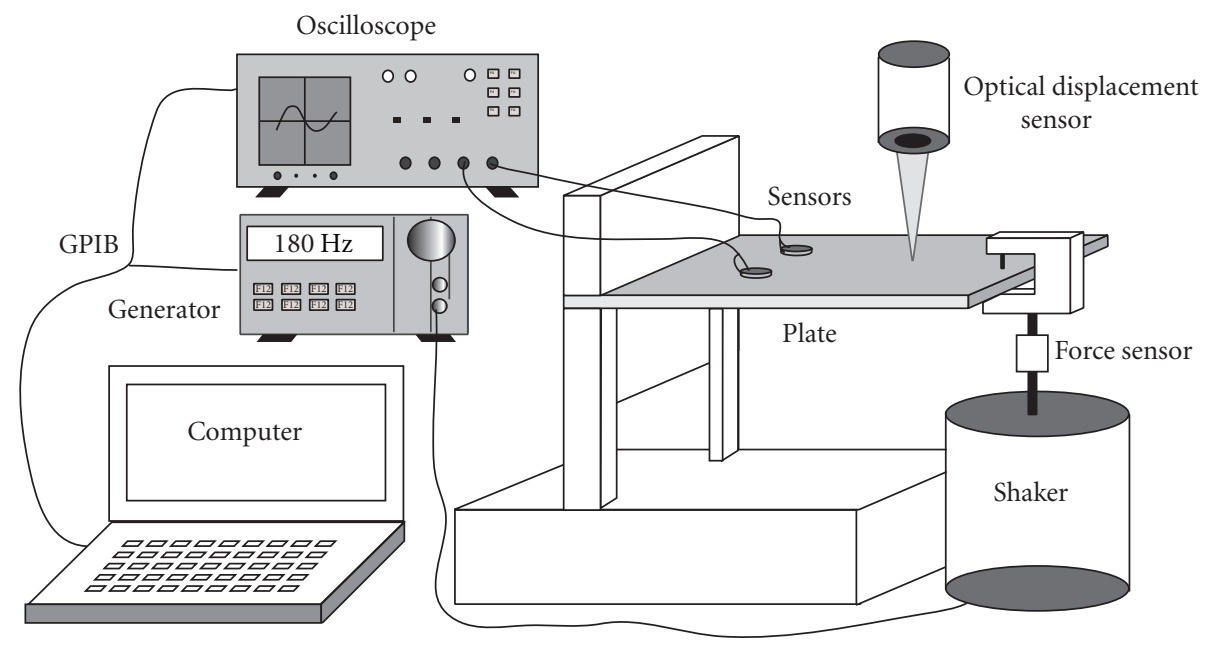

Figure 4: Experimental setup.

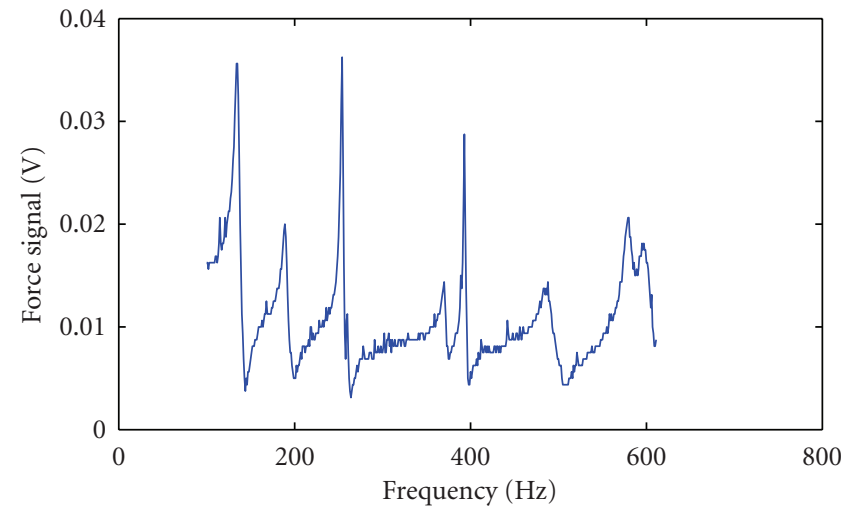

(a)

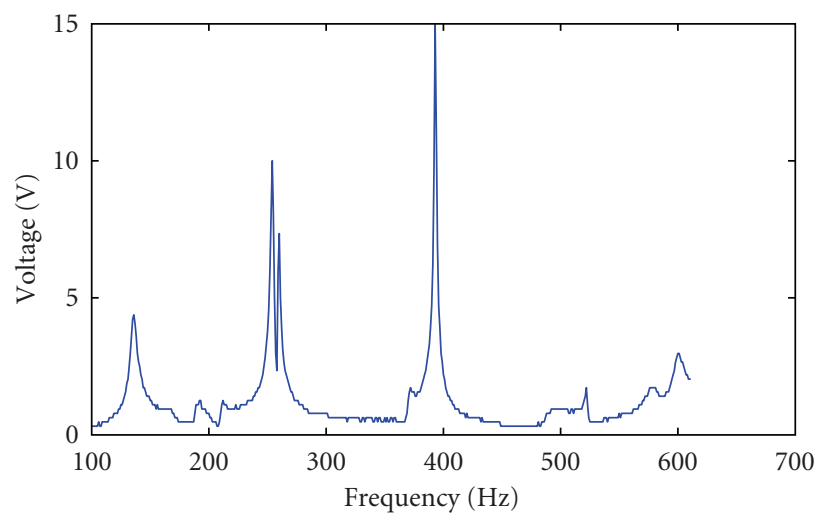

(c)

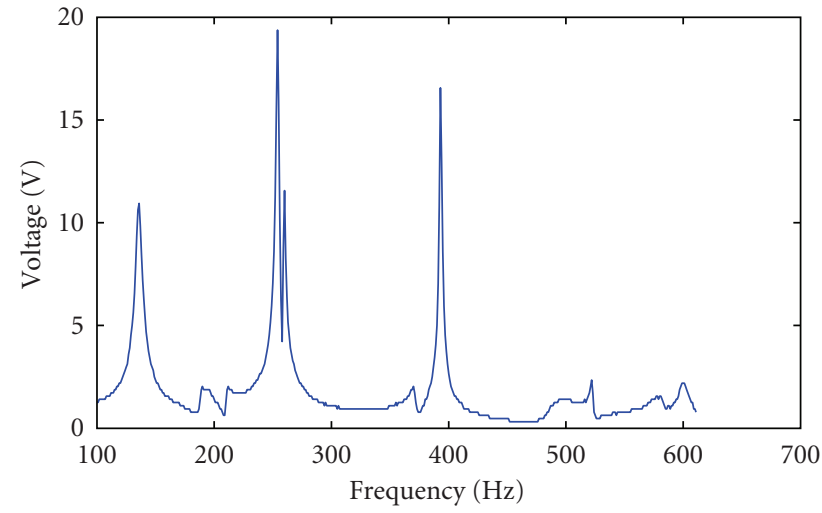

(b)

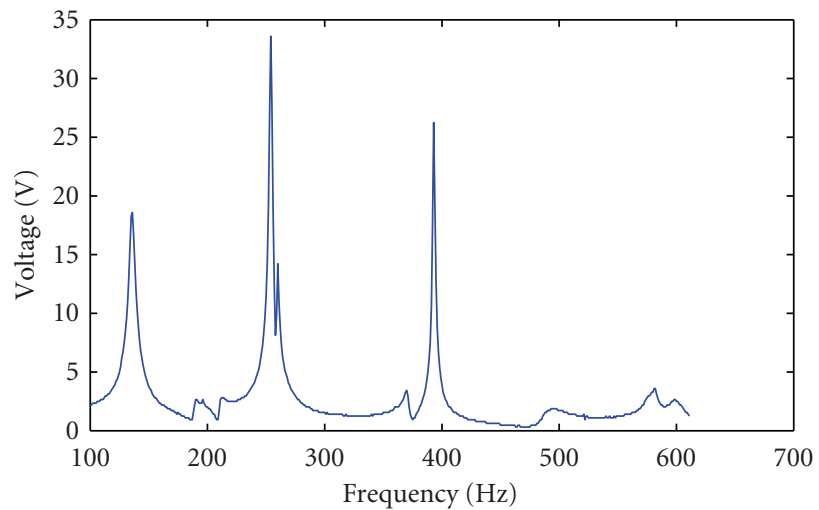

(d)

FIGURE 5: Force applied to the structure (a) and harvested electrical voltages for each sensor's location (b, c, and d).

1 or 2 millimetres have been chosen herein. They are all Pz27 discs from Ferroperm Piezoceramics A/S. The mechanical vibrations have been generated at the free end of the plate by an electromechanical shaker (Signal Force from Data Physics).

Different sensors have been used to measure the mechanical power applied to the structure. A force sensor from $\mathrm{PCB}$
Piezotronic embedded inside the grip has been used to measure the force applied to the plate. Its sensitivity is $2.3 \mathrm{mV} / \mathrm{N}$. An optical displacement sensor from Keyence has also been used to measure the deflection of the plate. Both signals provided by the sensors are recorded using an HP oscilloscope linked to a PC via a GPIB interface. The complete setup is presented in Figure 4. 
2.2. Low-Frequency Harmonic Investigation. In order to check the bandwidth capabilities of the harvester, a harmonic excitation has been performed. Its frequency range was defined from $100 \mathrm{~Hz}$ to $612 \mathrm{~Hz}$ with a $1 \mathrm{~Hz}$ resolution. A sinusoidal wave with the defined frequency and amplitude of $3 \mathrm{~V}$ was sent by the waveform generator to the shaker. This harmonic excitation was controlled by a Matlab programme. At each frequency step, the programme measured and plotted the maximum amplitude of the harvester response, the force applied to the plate, and its subsequent displacement versus the frequency, evaluated nearby the location of the shaker grip. This measurement allows the determination of the mechanical power applied to the structure.

Figure 5 presents the force applied to the plate and the voltage response of the SHM/energy harvester sensors. The previously defined conversion rate gives a typical value for the force measurement of $16 \mathrm{~N}$ at $136 \mathrm{~Hz}$.

These figures show a similitude between the force applied to the structure and the voltage response of the sensors. These peaks must correspond to the natural modes of the plate and are effectively detected by the SHM energy harvester. A difference of amplitude is noted between peaks depending on the frequency. A first reason may come from the original difference in the force applied to the structure. Moreover, at these frequencies, the sensors can be located at a minimum of deformation on the structure. As an example, at $189 \mathrm{~Hz}$ and $370 \mathrm{~Hz}$, the voltage response is present but with a low level. Nevertheless, this measurement shows the ability of SHM sensor to exhibit voltage at several frequencies, in this particular case from $100 \mathrm{~Hz}$ to $600 \mathrm{~Hz}$. A numerical model will be subsequently used to obtain a comprehension of these phenomena. As explained in Section 2.1, to be able to measure the mechanical power applied to the structure, the signal provided by the optical displacement sensor has been recorded for each frequency nearby the application of the force. Figure 6 shows a typical recorded signal which allows the evaluation of the displacement peak value of the plate.

This measurement gives a $20 \mu \mathrm{m}$ peak to peak displacement for the free edge of the plate when the frequency of the exciting signal for the shaker is near a natural mode of $387 \mathrm{~Hz}$. This frequency gives a maximum of voltage for each harvester as shown in Figure 5.

The following paragraph provides a comparison between the experimental results and the numerical simulations.

\section{Numerical Modelling}

The FEM model allows the computation of several physical data involved in the energy harvesting process. First, the displacement field of the host structure has been measured. Then, the voltage responses of the different sensors have been computed. These data will allow a better understanding of the energy harvesting process.

\subsection{FEM Modelling of the Structure under Harmonic Excita-} tion. The aluminium plate instrumented with piezoelectric SHM energy harvesters has been modelled using the software Comsol Multiphysics 4.2.

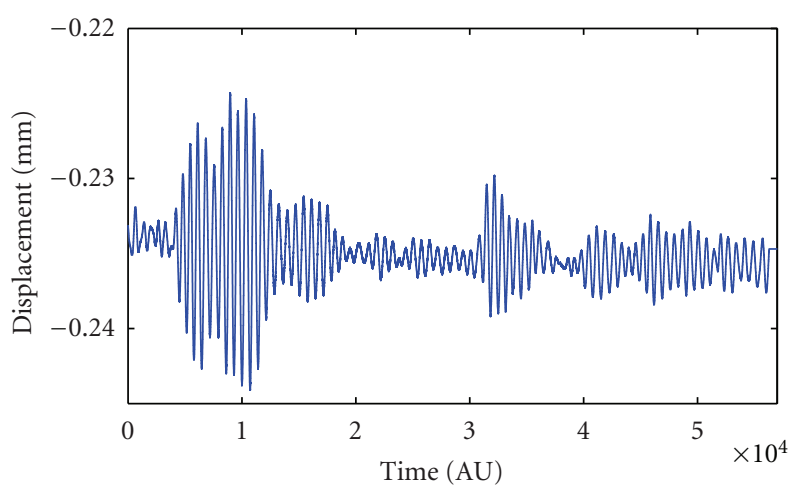

Figure 6: Optical displacement sensor output signal $(f=387 \mathrm{~Hz})$.

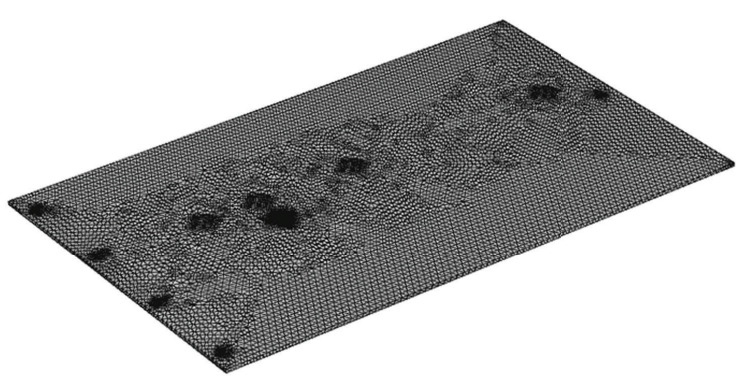

FIGURE 7: Harvesters' location and mesh of the plate.

The shaker is modelled as the actual input force presented in Figure 5. This force is applied on a circular area corresponding to the contact between the shaker grip and the structure.

The meshing is composed of 25253 tetrahedral elements. The meshing has been refined near the piezoelectric energy harvester as shown in Figure 7. Note that the meshing is voluntarily finer than the criterion in $\lambda / 8, \lambda$ being the wavelength of the deformation.

The working frequencies have been chosen in the $100 \mathrm{~Hz}$ to $612 \mathrm{~Hz}$ range with a $1 \mathrm{~Hz}$ step to generate different kinds of natural mode of the structure. Figure 8 gives the representation of the different modes at the previously measured natural frequencies. One can see the importance of sensors location since several areas correspond to nodes of displacement while others are antinodes of displacement.

The voltage response of the SHM sensors obtained running the FEM model will be presented in the following part.

3.2. Harvested Voltage Modelling using a FEM Model and Experimental Comparison. Running the FE model directly provides the sensor voltage response as shown in Figure 9. Their responses have been recorded around the resonant frequencies identified in Figure 5. One can see that the frequency behaviours of the sensors are correctly modelled. One obtains the same peak frequency and the voltage amplitude is also close to the experimental results. 


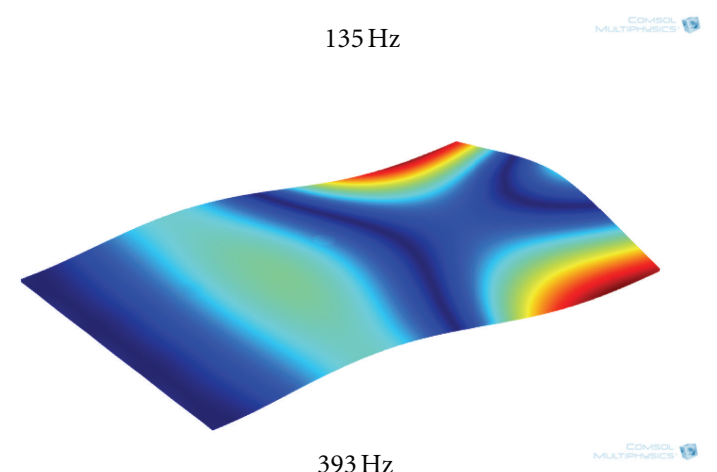

$393 \mathrm{~Hz}$

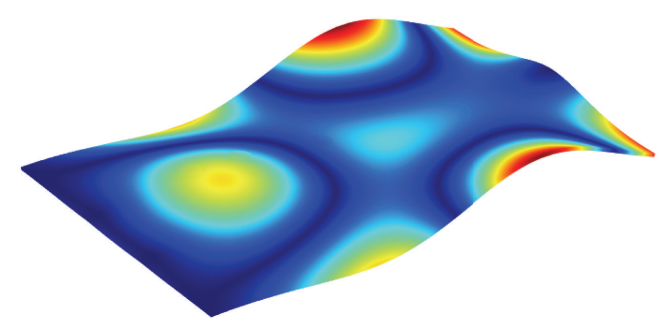

$254 \mathrm{~Hz}$

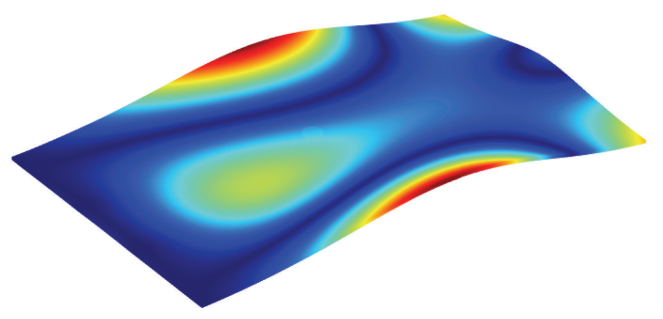

$587 \mathrm{~Hz}$

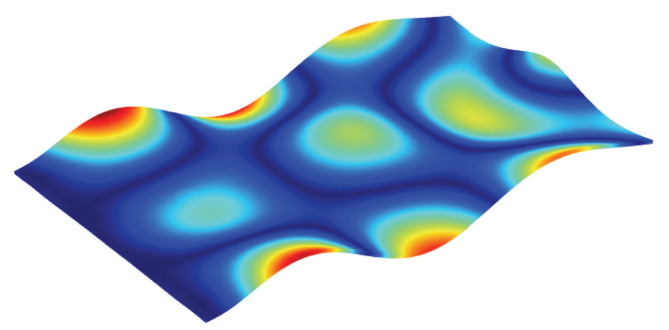

Figure 8: Plate deformation at various frequencies.

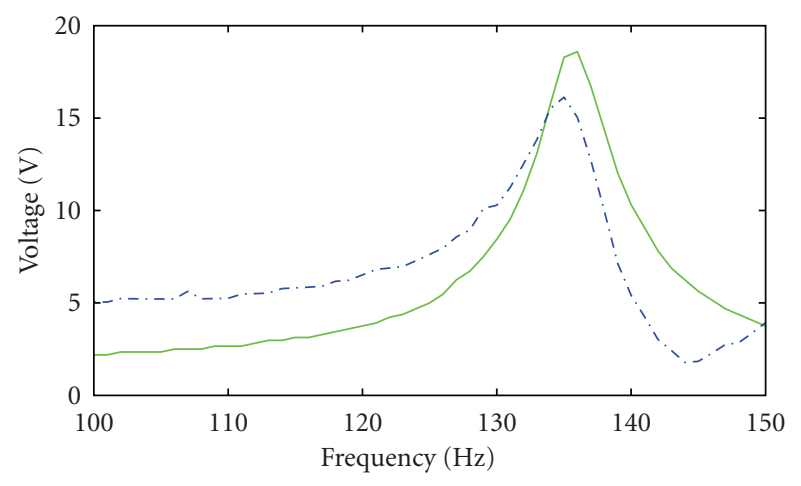

(a)

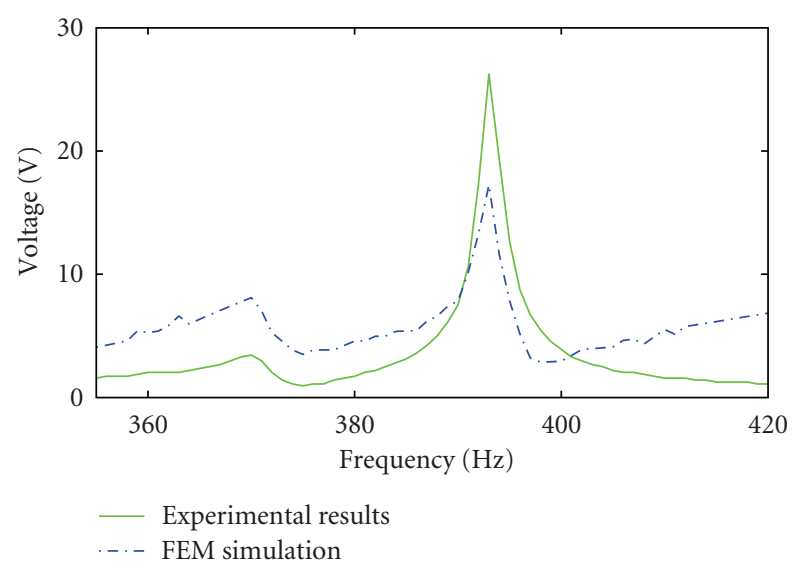

(c)

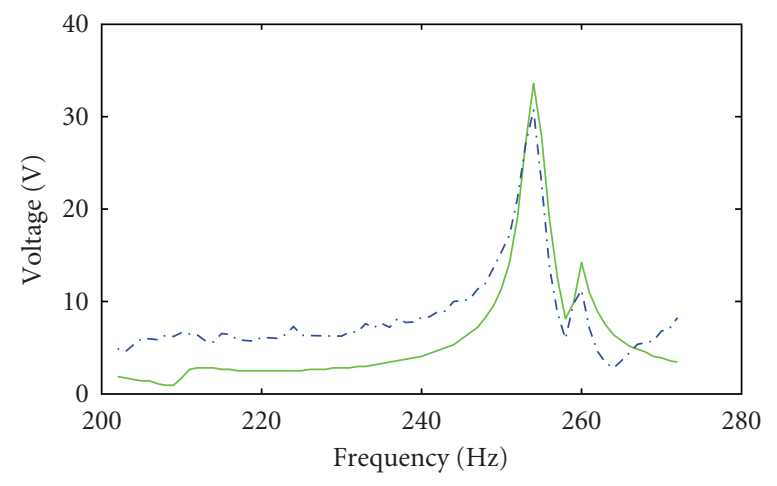

(b)

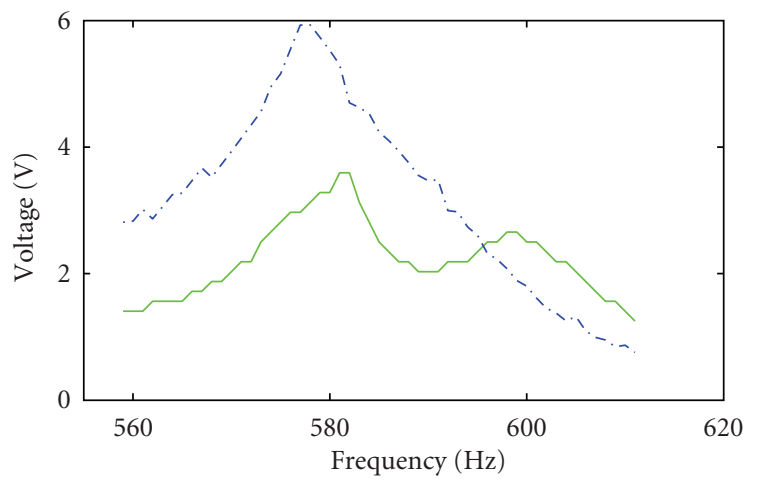

_ Experimental results

-.- FEM simulation

(d)

FIGURE 9: Experimental-numerical comparison of the harvested voltage. (a) $135 \mathrm{~Hz}$, (b) $254 \mathrm{~Hz}$, (c) $393 \mathrm{~Hz}$, and (d) $587 \mathrm{~Hz}$. 
TABLE 1: Experimental/FEM mechanical parameters.

\begin{tabular}{lccc}
\hline Resonant frequency $(\mathrm{Hz})$ & 135 & 252 & 387 \\
\hline $\begin{array}{l}\text { Force }(\mathrm{N}) \\
\begin{array}{l}\text { Peak displacement }(\mu \mathrm{m}) \\
\text { experimentation/FEM }\end{array}\end{array}$ & 16 & 15.54 & 12.4 \\
$\begin{array}{l}\text { Mechanical power }(\mathrm{mW}) \\
\text { experimentation/FEM }\end{array}$ & $\mathrm{NA} / 1357$ & $8 / 7.6$ & $10 / 9.6$ \\
\hline
\end{tabular}

The SHM energy harvesters actually measure resonance of the host structure and this independently of the frequency. This confirms the ability of SHM energy harvester to work over an extended frequency range. Concerning the difference of amplitude depending on the resonant frequencies, the FEM model keeps the ratio between the force applied at a given frequency and the subsequent voltage response.

\section{Power Harvesting Efficiency Evaluation}

A Linear technology component (LTC 3588) has been used to measure the power harvested and to form a complete energy harvesting solution optimized for high output impedance energy sources as piezoelectric transducers. The LTC 3588 integrates a low-loss full-wave bridge rectifier with a high efficiency bulk converter [17]. It provides an ultralow quiescent current under voltage lockout mode with a wide hysteresis window allowing charge to accumulate on an input capacitor until the buck converter can efficiently transfer a portion of the stored charge to the output. In regulation mode, the LTC 3588 enters a sleep state in which both input and output quiescent currents are minimal. The buck converter turns on and off as needed to maintain regulation. Four output voltages are available with up to $100 \mathrm{~mA}$ of continuous output current.

Using such a component, one can estimate the available electrical power for each sensor. This power is obtained for the minimum output load resistor providing a continuous output current at the selected voltage. The input stage of this component is fitted with a $18 \mathrm{~V}$ Zener diode in order to avoid overvoltage phenomenon. This configuration involves that the measurement of the electrical available power may be disturbed by the input stage of the components. As a matter of fact, if the harvested voltage is greater than the Zener voltage, a part of the harvested power is lost at the input stage.

For each natural resonant frequency, the different values for the experimental force applied to the structure and the displacement of the plate have been measured. The mechanical power has been subsequently calculated for each frequency step. The same calculations have been performed using the FEM model. The comparison is presented in Table 1.

The measurement of displacement at $135 \mathrm{~Hz}$ has not been performed since the displacements were out of range of the optical laser. This measurement is referred to as nonapplicable (NA) in Table 1.

A power estimation has also been carried out. At $387 \mathrm{~Hz}$, $27 \mathrm{~V}$ was provided by the SHM energy harvester, leading to $589 \mu \mathrm{W}$ transferred to a $22 \mathrm{k} \Omega$ load.
The different values obtained for the harvesting power clearly show that the classical Structural Health Monitoring ultrasonic sensors may be used in a harvesting process when they are not used for any monitoring tasks. The power harvested with these sensors is in the similar order than those obtained with cantilever beams in other works [5].

\section{Conclusions}

The aim of this present paper was to evaluate the capabilities of classical SHM sensors to carry out power harvesting from mechanical vibrations.

An experimentation phase showed that the sensors effectively work over an extended frequency range. The FEM model confirmed that the sensors actually respond to the different natural modes of the structure they are bonded onto. The power measurement showed that in this configuration, small PZT disc can harvest up to $589 \mu \mathrm{W}$, which is in the similar order than cantilever beams. Consequently, the SHM sensors appear to be a promising candidate to carry out power harvesting from mechanical vibrations. Since the monitoring process is a minor part of the functioning time, the idea is to use the SHM sensors as harvesting devices during the remaining time.

The future objectives are to develop a complete model of the energy harvesting process, from the initial mechanical vibration to the power transferred to a load.

\section{Acknowledgment}

The authors would like to thank Associated Professor S. Godts for his help in the development of a part of the theoretical modelling aspects of this work.

\section{References}

[1] D. Samson, M. Kluge, T. Becker, and U. Schmid, "Energy harvesting for autonomous wireless sensor nodes in aircraft," Procedia Engineering, vol. 5, pp. 1160-1163, 2010.

[2] M. Al Ahmad and H. N. Alshareef, "Energy harvesting from radio frequency propagation using piezoelectric cantilevers," Solid-State Electronics, vol. 68, pp. 13-17, 2012.

[3] L. Chen, F. L. Ponta, and L. I. Lago, "Perspectives on innovative concepts in wind-power generation," Energy for Sustainable Development, vol. 15, no. 4, pp. 398-410, 2011.

[4] H. Sodano, E. A. Magliula, G. Park, and D. J. Inman, "Electric power generation using piezoelectric devices," in Proceedings of the 13th International Conference on Adaptive Structures and Technologies, Potsdam, Germany, 2002.

[5] M. Ferrari, V. Ferrari, M. Guizzetti, D. Marioli, and A. Taroni, "Piezoelectric multifrequency energy converter for power harvesting in autonomous microsystems," Sensors and Actuators A, vol. 142, no. 1, pp. 329-335, 2008.

[6] Y. Hu, T. Hu, and Q. Jiang, "Coupled analysis for the harvesting structure and the modulating circuit in a piezoelectric bimorph energy harvester," Acta Mechanica Solida Sinica, vol. 20, no. 4, pp. 296-308, 2007.

[7] M. Zhu and S. Edkins, "Analytical modelling results of piezoelectric energy harvesting devices for self-power 
sensors/sensor networks in structural health monitoring," Procedia Engineering, vol. 25, pp. 195-198, 2011.

[8] E. Lefeuvre, A. Badel, C. Richard, L. Petit, and D. Guyomar, "A comparison between several vibration-powered piezoelectric generators for standalone systems," Sensors and Actuators A, vol. 126, no. 2, pp. 405-416, 2006.

[9] S. Grondel, C. Paget, C. Delebarre, J. Assaad, and K. Levin, "Design of optimal configuration for generating A0 Lamb mode in a composite plate using piezoceramic transducers," Journal of the Acoustical Society of America, vol. 112, no. 1, pp. 84-90, 2002.

[10] C. A. Paget, K. Atherton, and E. W. O'Brien, “Triangulation algorithm for damage location in aeronautical composite structures," in Proceedings of the 8th International Workshop on Structural Health Monitoring, pp. 363-370, Stanford, Calif, USA, 2003.

[11] C. A. Paget, K. Tiplady, M. Kluge, T. Becker, and J. Schalk, "Feasibility study on wireless impact damage assessment system for thick aeronautical composites," in Proceedings of the 5th European Workshop on Structural Health Monitoring, pp. 125-130, Sorrento, Italy, 2010.

[12] F. Benmeddour, S. Grondel, J. Assaad, and E. Moulin, "Study of the fundamental Lamb modes interaction with asymmetrical discontinuities," NDT and E International, vol. 41, no. 5, pp. 330-340, 2008.

[13] F. Benmeddour, S. Grondel, J. Assaad, and E. Moulin, "Experimental study of the A0 and S0 Lamb waves interaction with symmetrical notches," Ultrasonics, vol. 49, no. 2, pp. 202-205, 2009.

[14] F. L. Di Scalea, H. Matt, and I. Bartoli, “The response of rectangular piezoelectric sensors to Rayleigh and Lamb ultrasonic waves," Journal of the Acoustical Society of America, vol. 121, no. 1, pp. 175-187, 2007.

[15] B. Chapuis, Contrôle Santé Intégré par méthode ultrasonore des réparations composites collées sur des structures métalliques, Ph.D. thesis, Université Denis Diderot, Paris, France, 2010.

[16] T. Sainthuile, C. Delebarre, S. Grondel, and C. Paget, "Bond Graph model of a SHM piezoelectric energy harvester," in Proceedings of the 8th International Workshop on Structural Health Monitoring, pp. 618-625, Stanford, Calif, USA, 2011.

[17] Linear Technology. LTC 3588 datasheet. 

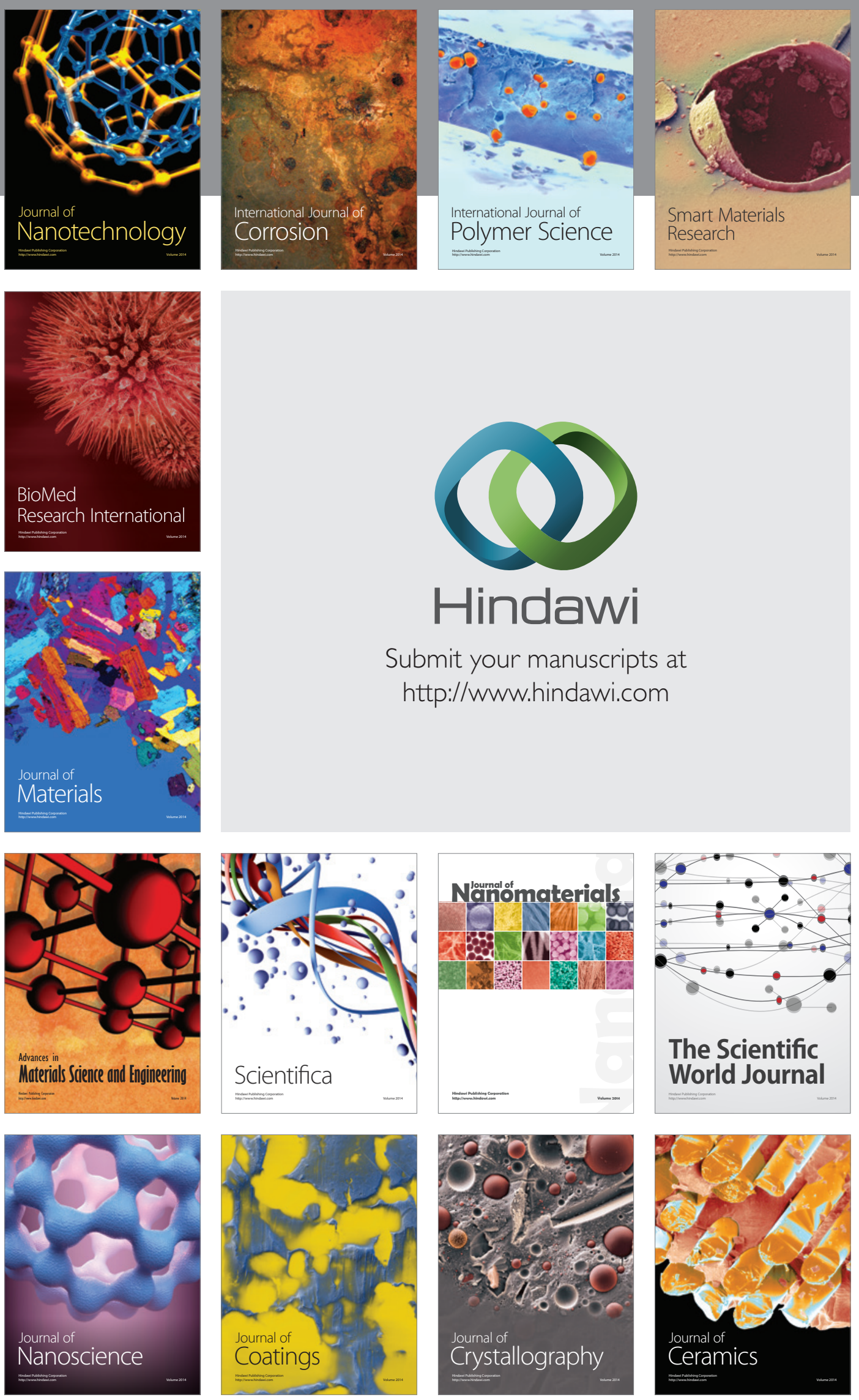

The Scientific World Journal

Submit your manuscripts at

http://www.hindawi.com

\section{World Journal}

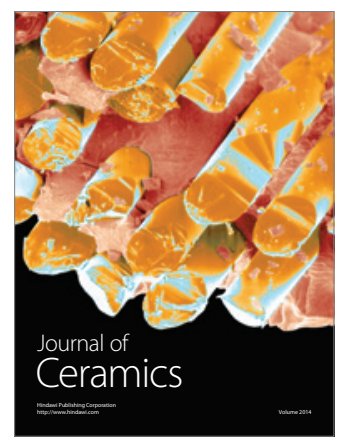

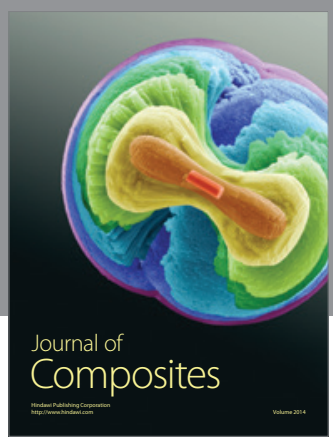
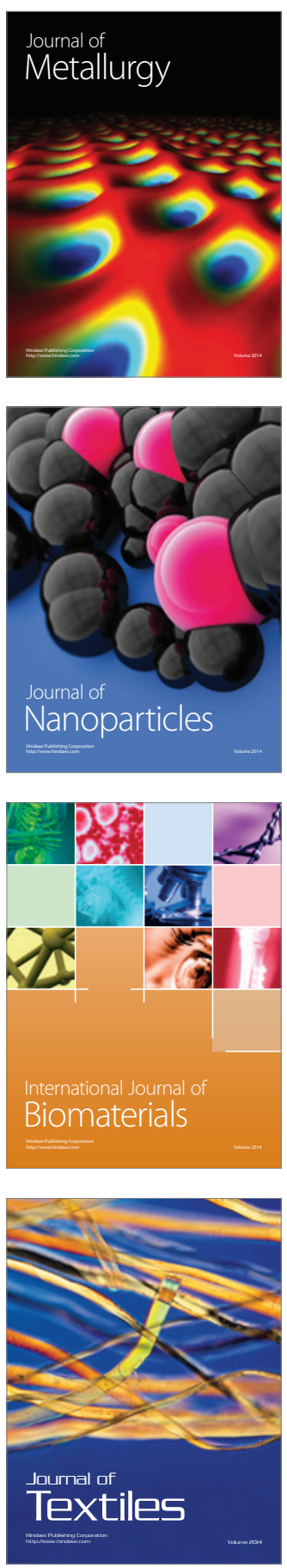